\title{
Extended methods using thick-targets for nuclear reaction data of radioactive isotopes
}

\author{
Shuichiro Ebata ${ }^{1, a}$, Masayuki Aikawa ${ }^{1}$, and Shotaro Imai ${ }^{2}$ \\ ${ }^{1}$ Faculty of Science, Hokkaido University, Sapporo 060-0810, Japan \\ ${ }^{2}$ Institute for the Advancement of Higher Education, Hokkaido University, Sapporo 060-0817, Japan
}

\begin{abstract}
The nuclear transmutation is a technology to dispose of radioactive wastes. However, we do not have enough basic data for its developments, such as thick-target yields (TTY) and the interaction cross sections for radioactive material. We suggest two methods to estimate the TTY using inverse kinematics and to obtain the excitation function of the interaction cross sections which is named the thick-target transmission (T3) method. We deduce the energy-dependent conversion relation between the TTYs of the original system and its inverse kinematics, which can be replaced to a constant coefficient in the high energy region. Furthermore we show the usefulness of the T3 method to investigate the excitation function of the ${ }^{12} \mathrm{C}+$ ${ }^{27} \mathrm{Al}$ reaction in the simulation.
\end{abstract}

\section{Introduction}

Nuclear data of radioactive isotopes (RI) are important for not only nuclear physics but also nuclear engineering. Nuclear transmutation of long-lived fission products (LLFP) is one of key technologies to dispose of radioactive wastes of which the data are also significant. However we do not have enough basic data related to radioactive targets such as the thick-target yields (TTY) which is necessary to investigate the reaction data with a lump target. Because the experiments with the targets including LLFP are difficult due to their radiation, chemical properties, etc. The excitation function of the interaction cross section is also necessary to evaluate the TTY microscopically. In these days, the progress of the RI beam technology allows us to measure the nuclear data in inverse kinematics without the LLFP targets.

We suggest two methods using a thick-target to obtain and to accumulate the nuclear data. One is a new method to estimate TTYs using inverse kinematics in a similar way as a cross section. Another new method to measure the excitation function of interaction cross sections without readjustments of beam settings, is derived from the ordinal transmission method with thick-targets.

In this work, we introduce our methods and show the results with using open simulation codes. The potential of the methods for LLFP lumps is also discussed.

\section{Methods}

We explain briefly our methods of the conversion method and the thick-target transmission (T3) method whose details are written in the Refs. [1] and [2].

\subsection{Conversion method between TTYs}

In order to measure the TTY of radioactive targets, we suggest the conversion method. This method is deduced from the relation between a forward reaction and the reaction of its inverse kinematics. At first, the differential reaction probability $d Y$ at an infinitesimal length in the target $d x$ can be written as,

$$
d Y=\sigma \frac{\rho_{T} N_{\mathrm{A}}}{A_{T}} d x,
$$

where $\sigma$ is the cross section $\left(\mathrm{cm}^{2}\right)$, the Avogadro constant $N_{\mathrm{A}}\left(\mathrm{mol}^{-1}\right)$, the density $\rho_{T}\left(\mathrm{~g} \mathrm{~cm}^{-3}\right)$ and the atomic mass in the target $A_{T}\left(\mathrm{~g} \mathrm{~mol}^{-1}\right)$. The TTY $Y(\varepsilon)$ is calculated using the (1) and the mass stopping power $S(\varepsilon)$,

$$
Y\left(\varepsilon_{i}\right)=\frac{N_{\mathrm{A}} A_{P}}{A_{T}} \int_{0}^{\varepsilon_{i}} \frac{\sigma(\varepsilon)}{S(\varepsilon)} d \varepsilon,
$$

where $S(\varepsilon)=-\frac{A_{P} d \varepsilon}{d\left(\rho_{T} x\right)}$, and $A_{P}$ is the atomic mass of the projectile. The $\varepsilon$ is an energy per nucleon $E / A_{P}$. The $Y(\varepsilon)$ is depending on the incident energy $\varepsilon_{i}$. It is assumed that the incident particles are stopped inside the target. The stopping power is well-studied and can be fitted by parameters in each reaction system, such as it is well reproduced by SRIM code [3].

Here we consider the inverse kinematics of the reaction system. In this work, the original reaction is named as "forward" in which the stopping power is denoted as $S_{\text {for }}(\varepsilon)$. Therefore in the inverse kinematics, that is denoted as $S_{\text {inv }}(\varepsilon)$. The TTY of the inverse kinematics is also described according to Eq. (2), although the atomic masses of projectile and target are changed and $S_{\text {inv }}(\varepsilon)$ is used. Because the $\sigma(\varepsilon)$ is common in both reaction systems, the conversion of derivatives of the TTY from the "inverse" to

\footnotetext{
a e-mail: ebata@nucl.sci.hokudai.ac.jp
} 
"forward" system is obtained by using the ratio between the stopping powers theoretically:

$$
R(\varepsilon)=\frac{d Y_{\mathrm{for}}}{d Y_{\mathrm{inv}}}=\frac{A_{P}^{2}}{A_{T}^{2}} \frac{S_{\mathrm{inv}}(\varepsilon)}{S_{\mathrm{for}}(\varepsilon)} .
$$

In a high energy reaction, we found that the conversion coefficient $R(\varepsilon)$ can be approximately replaced to a constant value $\tilde{R} ; d Y_{\text {for }}(\varepsilon) \simeq \widetilde{R} d Y_{\text {inv }}(\varepsilon)$. If a contribution of the low energy part is sufficiently small, we can use

$$
Y_{\mathrm{for}}(\varepsilon) \simeq \tilde{R} Y_{\mathrm{inv}}(\varepsilon) .
$$

The energy dependence of $R(\varepsilon)$ disappears in a high energy region, such as shown in Fig. 1. We test the availability of the replacement in Sect. 3.1.

\subsection{Thick-target transmission method}

The thick-target transmission (T3) method is deduced by an extension of the ordinal transmission method. When we measure the energy dependence of the cross section in the ordinal transmission method, it is necessary to adjust the beam for each energy. We consider a beam attenuation by nuclear reactions at a length $x(\mathrm{~cm})$ from the surface of a target. The numbers of incident particles and reacted particles at the $x$ are denoted as $N_{i}(x)$ and $N_{r}(x)$. Since $N_{i}(0)$ is constant which is sum of $N_{i}(x)$ and $N_{r}(x)$, the derivative $d N_{i}(x)$ corresponds to $-d N_{r}(x)$ which is the number of reacted particles in the $d x$, and is written as Eq. (1) with the interaction cross section $\sigma_{I}\left(\mathrm{~cm}^{2}\right)$ :

$$
-\frac{d N_{i}(x)}{N_{i}(x)}=\sigma_{I} \frac{\rho_{T} N_{\mathrm{A}}}{A_{T}} d x .
$$

Finally the beam attenuation can be obtained as,

$$
\begin{aligned}
N_{i}(L)= & N_{i}(0) e^{-Y(L)}, \\
& Y(L)=n_{T} \int_{0}^{L} \sigma_{I}(x) d x,
\end{aligned}
$$

where $L$ is the target thickness, $n_{T} \equiv \rho_{T} N_{\mathrm{A}} / A_{T}$ and the $Y(L)$ is the yield represented in the thickness $L$. If the target is significant thin, the $Y$ can be approximated as $Y \simeq n_{T} \sigma_{I} L$ which completely corresponds to that in the ordinal transmission method.

According to Eq. (5), the $\sigma_{I}(L)$ can be derived as

$$
\begin{aligned}
\sigma_{I}(L) & =-\frac{1}{n_{T}} \frac{Y(L+d L)-Y(L)}{L} \\
& =-\frac{1}{n_{T} d L} \ln \left[\frac{N_{i}(0)}{N_{i}(L)} \frac{N_{i}^{\prime}(L+d L)}{N_{i}^{\prime}(0)}\right],
\end{aligned}
$$

which corresponds to the result in the ordinal transmission method using the attenuated beam passing through $L \mathrm{~cm}$ in the target with a thickness $d L$. The $N_{i}$ and $N_{i}^{\prime}$ mean the particle numbers in different experiment runs with targets with different thickness. Here the $L$ can be transformed into $\varepsilon$ using the stopping power:

$$
\varepsilon(L)=\varepsilon(0)-\frac{\rho_{T}}{A_{P}} \int_{0}^{L} S(x) d x .
$$

Finally the $\sigma_{I}(\varepsilon)$ can be obtained with a changing target thickness instead of beam adjustments.

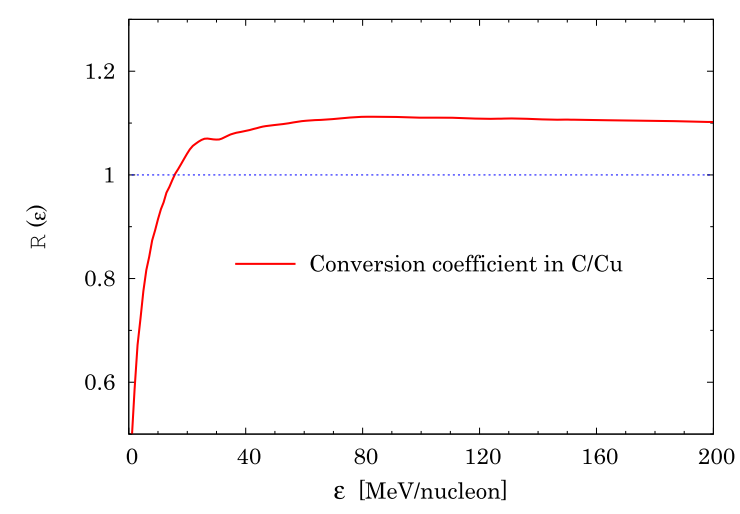

Figure 1. Energy dependence of conversion coefficient shown in Eq. (3). The stopping powers are evaluated by SRIM2008 [3].

Table 1. TTYs in the "forward" reactions at 40 and $100 \mathrm{MeV} /$ nucleon derived from Eqs. (2) and (4).

\begin{tabular}{ccc}
\hline & $Y_{\text {for }}(40)$ & $Y_{\text {for }}(100)$ \\
\hline Eq. (2) & $0.91 \times 10^{-5}$ & $0.114 \times 10^{-3}$ \\
Eq. (4) & $0.94 \times 10^{-5}$ & $0.113 \times 10^{-3}$ \\
\hline
\end{tabular}

\section{Results}

In this section, we introduce simulation results by SRIM and PHITS [4] codes, according to the conversion method and the T3 method to test their availability. Furthermore we mention a potential of the combination of these methods.

\subsection{Deduced TTY from the inverse kinematics}

To test the simplified conversion method Eq. (4), we show the examples of the ${ }^{\text {nat }} \mathrm{Cu}\left({ }^{12} \mathrm{C}, \mathrm{X}\right){ }^{24} \mathrm{Na}$ reaction [5] in which there are the data in a low energy region, although it is a rare reaction event. When we use the Eq. (3) to convert $Y_{\text {inv }}(\varepsilon)$ into $Y_{\text {for }}(\varepsilon)$, it is completely correct due to the definition. Of course the Eq. (3) can be used only when the $\sigma$ is known in all energy range. The simplified conversion Eq. (4) is required because it is so difficult to prepare the completed $\sigma$. Figure 1 shows the $R(\varepsilon)$ using the $S_{\text {for }}(\varepsilon)$ and $S_{\text {inv }}(\varepsilon)$ of each systems which are evaluated by SRIM2008 code [3]. We can see the conversion of $R(\varepsilon)$ approaching to a constant value $(\tilde{R} \sim 1.1)$ at the high energy over $50 \mathrm{MeV} /$ nucleon.

We compare the results calculated with Eqs. (2) and (4) which are summarized in Table 1. The constant conversion relation Eq. (4) is effective in practical ways, although the results have small differences.

\subsection{Excitation function of the $\sigma_{I}$}

We test the T3 method to confirm whether the results in the ordinal transmission method can be reproduced or not, using a virtual experiments $\left({ }^{12} \mathrm{C}+{ }^{27} \mathrm{Al}\right)$ simulated by a Monte Carlo simulation code PHITS [4]. The simulation parameters are listed in Table 2. Figure 2 shows the ${ }^{12} \mathrm{C}$ beam attenuation (circle) and energy (cross) of the beam as a function of the $L$.

According to Eq. (6), we can evaluate $\sigma_{I}(\varepsilon)$ with the beam attenuation and particle energies plotted in Fig. 2. The $\sigma_{I}(\varepsilon)$ are shown in Fig. 3 with those by the transmission method (dashed line) and experimental data $[6,7]$ (triangle and square). The $\sigma_{I}(\varepsilon)$ of the T3 method 
Table 2. Simulation parameters in PHITS.

\begin{aligned} & \hline Energy: $100 \mathrm{MeV} /$ nucleon \\ & Target: ${ }^{27} \mathrm{Al} \\ &$ Density: $2.7 \mathrm{~g} / \mathrm{cm}^{3} \\ &$ Thickness: $1.22 \mathrm{~cm} \\ &$ dL: $0.1 \mathrm{~cm}(0<\mathrm{L}<1.0 \mathrm{~cm}) \\ & 0.02 \mathrm{~cm}(1.0<\mathrm{L}<1.22 \mathrm{~cm}) \\ &$ Trial numbers: $10^{5} \\ &$\hline\end{aligned}

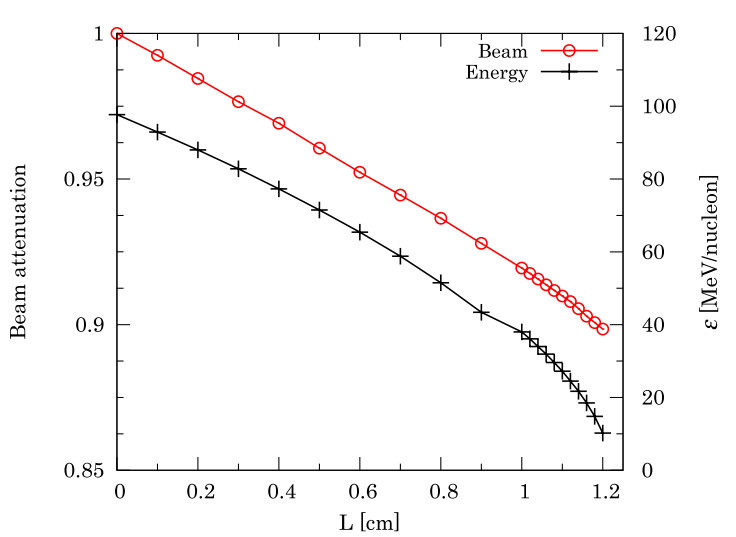

Figure 2. Beam attenuation and particle energy with respect to target thickness $L$.

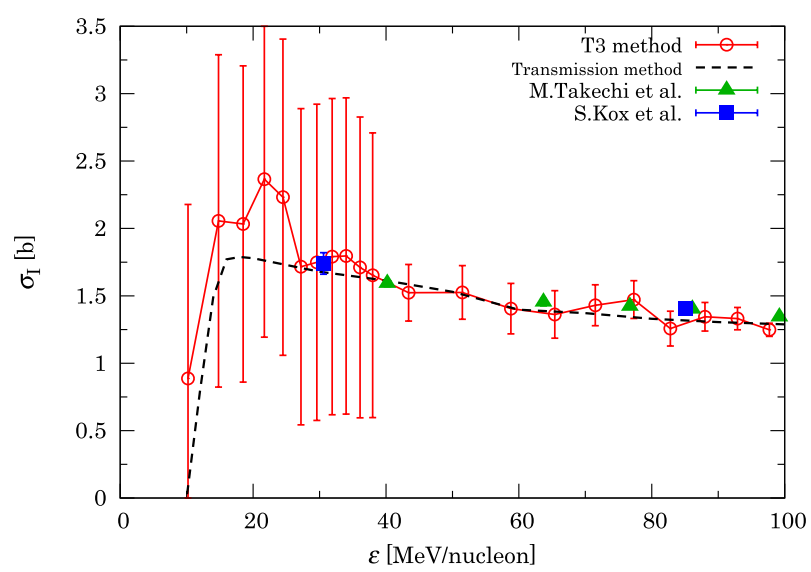

Figure 3. The interaction cross section $\sigma_{I}(\varepsilon)$; T3 method (circle), transmission method (dashed line) and experimental data (triangle and square) [6,7].

well reproduce others, although they have large errors in the low energy region, due to the small statistics.

We mention the combination of the conversion method and the T3 method. To measure the TTY with radioactive target, the conversion method is not always sufficient, since it has a restriction. If the residual nuclide on this reaction decays without gamma rays, its amount can not be counted, which is similar to the restriction of the activation method. The T3 method is independent from the kinds of nuclides. The interaction cross section is the probability to break projectiles, namely it corresponds to the transmutation reaction cross section. We expect that the combination might realize the accumulation of the data (TTY and $\sigma_{I}(\varepsilon)$ ) with any radioactive targets.

\section{Summary and perspective}

We introduce two methods to measure the data related to RI using the thick targets, which is to avoid the experiments with radioactive targets.

One is the conversion method which can derive the TTY taking advantages of the inverse kinematics. Although the conversion relation has basically an energy dependence, we found a more simplified conversion relation in a high energy region.

The other is the T3 method which is the extended transmission method with thick-targets. In the T3 method, the excitation function of $\sigma_{I}$ can be measured by changing the target thickness instead of the beam adjustment.

We mention the possibility to use the combination for the measurement of the transmutation reaction data with LLFP targets.

We will apply the methods to the reaction system with stable targets and the beams of LLFPs $\left({ }^{79} \mathrm{Se},{ }^{93} \mathrm{Zr}\right.$, ${ }^{107} \mathrm{Pd},{ }^{135} \mathrm{Cs}$, etc.). These applications will provide and accumulate useful data.

This work was funded by ImPACT Program of Council for Science, Technology and Innovation (Cabinet Office, Government of Japan).

\section{References}

[1] M. Aikawa, S. Ebata and S. Imai, Nucl. Instr. Meth. B 353, 1 (2015)

[2] M. Aikawa, S. Ebata and S. Imai, Nucl. Instr. Meth. B 383, 156 (2016)

[3] J. Ziegler, J. P. Biersack, M. Ziegler, SRIM: the Stopping and Range of Ions in Matter, http://www.srim.org

[4] T. Sato et al., J. Nucl. Sci. Technol. 50, 913 (2013)

[5] H. Yashima et al., Phys. Rev. C 66, 044607 (2002)

[6] S. Kox et al., Phys. Lett. B 159, 15 (1985)

[7] M. Takechi et al., Phys. Rev. C 79, 061601 (2009) 